\title{
The Mathematics Division of the National Center for Theoretical Sciences
}

\author{
by Wen-Ching W. Li
}

\section{Introduction}

National Center for Theoretical Science (NCTS) in Taiwan was established on August 1, 1997 fully funded by the National Science Council (NSC) in Taiwan. (NSC will become the Ministry of Science and Technology as of 2014.) The Center is hosted in Hsinchu jointly by $\mathrm{Na}$ tional Tsing Hua University (NTHU) and National Chiao Tung University (NCTU). The first funding phase was six years (1997-2003). At the beginning of the second phase (2004-2008), an administrative decision was made by NSC to expand the structure of the NCTS by adding two subprojects: Subproject-I in Taipei hosted by National Taiwan University (NTU) and Subproject-II in Tainan

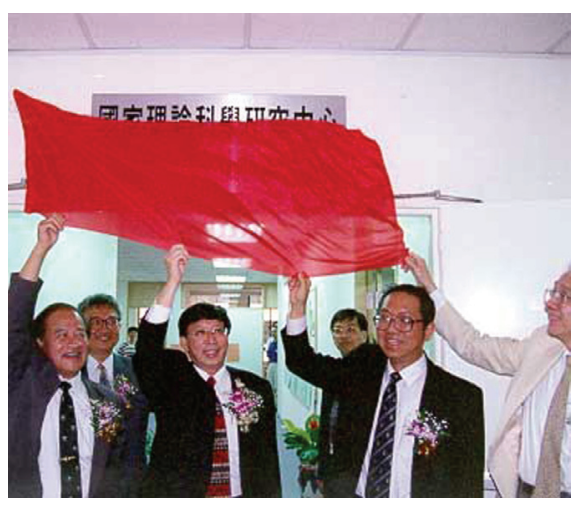

NCTS inauguration 1977

hosted by National Cheng Kung University (NCKU) and National Sun Yet San University (NSYSU) while the headquarter remains in Hsinchu. National Central University (NCU) joined NTHU and NCTU to become one of the hosting institutions in 2009, the beginning of the third phase (2009-2014).
The Center consists of two separate, equally funded divisions, Mathematics and Theoretical Physics. The total budget for 2013 is 75 million NT dollars. Wen-Ching Winnie Li of Penn State University and NTHU is the current NCTS director, who also serves as the head of the Mathematics Division. She started this position in 2009, succeeding Jing Yu. XiaoGang He of NTU and NTHU is the

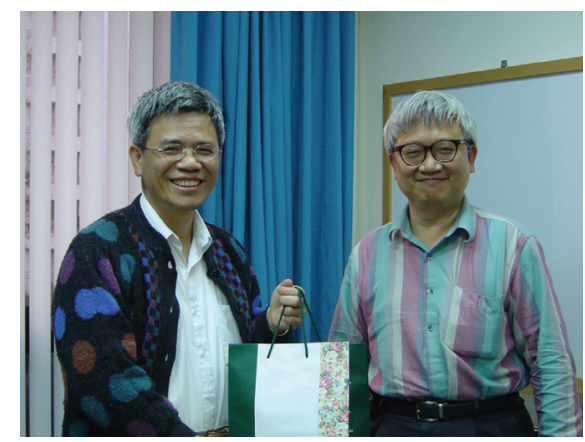

past directors C.-S. Lin and J. Yu

head of the Physics Division. Each subproject also has two divisions; Jung-Kai Chen of NTU and Ching-Hung Lam of Academia Sinica (AS) are currently in charge of the mathematics division in Sub-project-I and Subproject II, respectively. In the history of NCTS, the mathematics division has had three division heads, who also served as directors of the center: Chang-Shou Lin (now at NTU), Jing Yu (now at NTU) and Wen-Ching Li.

The missions of NCTS are:

1. To foster world class outstanding researchers, and attract top young researchers to do cutting edge research in theoretical sciences;

2. To attract worldwide outstanding theoretical scientists to do research in Taiwan;
3. To develop inter-disciplinary and cross-area scientific research programs;

4. To promote international cooperation and collaborations, aiming to become a leading research institution in Asia and in the world.

\section{Administrative Structure}

In the Mathematics Division, policies and decisions are made by two committees. The program committee is concerned with the scientific activities of the center, and the executive committee makes decisions on policies and hiring. Currently, program committee members are Gerard Jennhwa Chang (discrete mathematics) from NTU, Jungkai

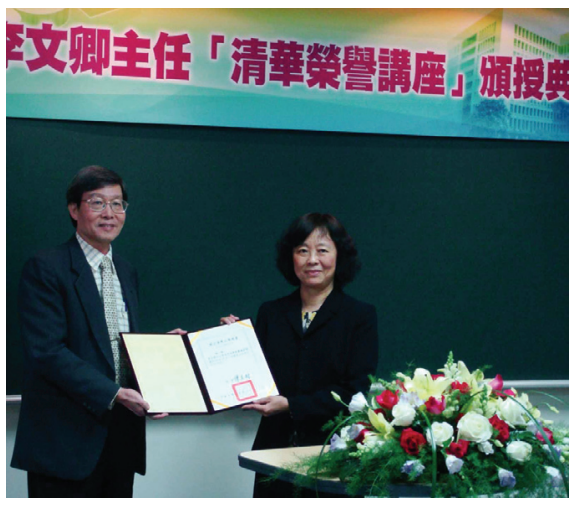

current director W.-C. $\mathrm{Li}$

Chen (algebraic geometry) from NTU, Jih-Hsin Cheng (differential geometry and geometric analysis) from AS, Ching-Hung Lam (vertex operator algebra and representation theory) of AS, Sze-Bi Hsu (dynamical systems and mathematical biology) of NTHU, Wen-Ching Li (number theory) of NCTS, Tai-Chia Lin (nonlinear PDE, math physics) of NTU, Wen-Wei Lin (scien-tific computation) of NCTU, 
Shuenn-Jyi Sheu (probability theory) of NCTU, Chih-Wen Shih (dynamical systems and math biology) from NCTU, Chiung-Jue Sung (differential geometry and geometric analysis) from NTHU, Wei-Cheng Wang (scientific computation) of NTHU, Eugene Zhu Xia (algebraic geometry) from NCKU, Yifan Yang (number theory) from NCTU, and Chia-Fu Yu (arith-

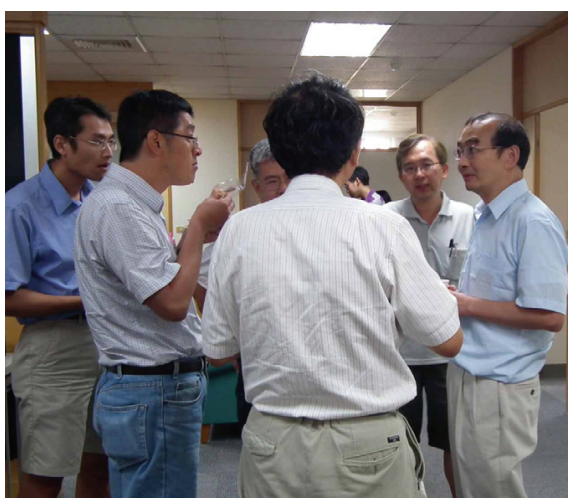

tea time

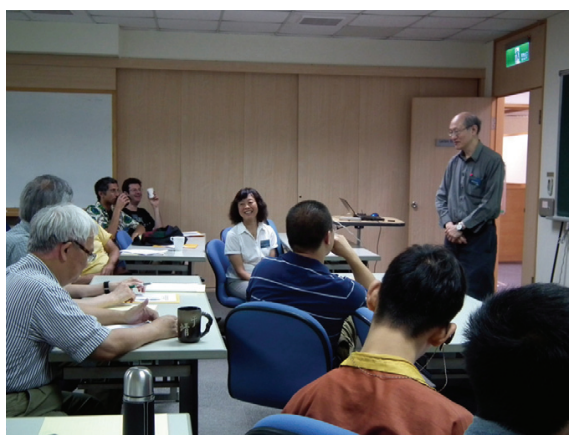

waiting to start a lecture

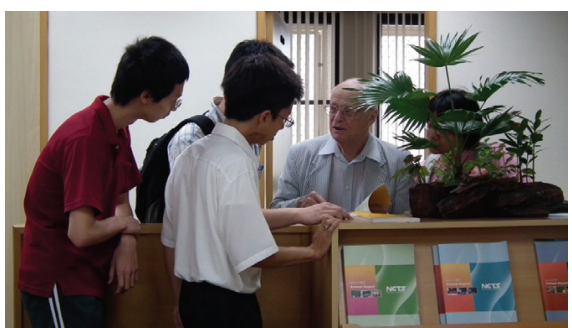

Serre talking to students

metic geometry) from AS. The executive committee consists of Chiun-Chuan Chen (nonlinear PDE) of NTU, Jung-Kai Chen (algebraic geometry) of NTU, Sze-Bi Hsu (dynamical systems and mathematical biology) of NTHU, Ching-Hung Lam (vertex operator algebra) of AS, Wen-Ching Li (number theory) of
NCTS, Shuenn-Jyi Sheu (probability theory) of NCU, and Yifan Yang (number theory) of NCTU.

The International Scientific Advisory Committee (ISAC) is formed to give advice and guidance of the future direction of the Mathematics Division. ISAC committee members are Ching-Li Chai (University of Pennsylvania and Academia Sinica), Bernold Fiedler (Free University of Berlin), Thomas Yizhao Hou (Cal Tech), Ker-Chau Li (UCLA), Shigefumi Mori (RIMS, Kyoto University), Richard Schoen (Stanford University), Horng-Tzer Yau (Harvard University), Shing-Tung Yau (Harvard University), and Shouwu Zhang (Princeton University).

\section{Scientific Staff}

Modeled after MSRI at Berkeley, NCTS has no permanent scientists. Each year the center invites numerous short- and mid-term visitors. From time to time, there are long term visitors. For example, Der-Chen Chang of Georgetown University spent his sabbatical year visiting NCTS (Dec 2012-July 2013). The long term scientific research personnel in Mathematics Division consist of center scientists, postdoctoral fellows, and research associates.

Center Scientists are responsible for organizing activities for the center. In return for their service, their teaching loads in their home institutions are reduced. Current Center Scientists in Hsinchu area are: Der-Chen Chang (geometry, PDE and harmonic analysis) from Georgetown University, Chieh-Yu Chang (number theory) from NTHU, Kuan-Yu Chen (probability theory) from NCTU, Hung-Lin Chiu (differential geometry and geometric analysis) from NCU, Chia-Chieh Jay Chu (scientific computation) from NTHU, Sze-Bi Hsu (dynamical systems and mathematical biology) from NTHU, Wen-Ching W. Li (number theory and applications) from Penn State University and NCTS, Wen-Wei Lin (scientific computation) from NCTU, Chih-Wen Shih (dynamical systems) from NCTU, and
Shuenn-Jyi Sheu (probability theory) from NCU. Ming-Lun Hsieh (number theory) from NTU and Jeng-Daw Yu

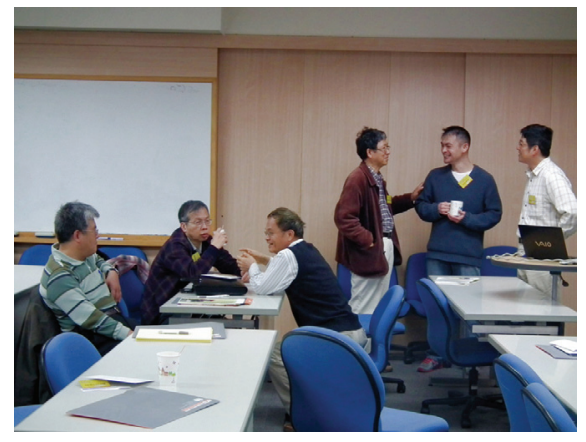

schmoozing with friends

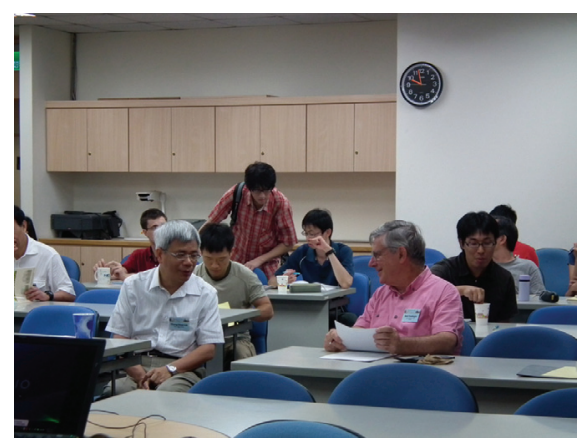

C.S.Lin and N. Trudinger

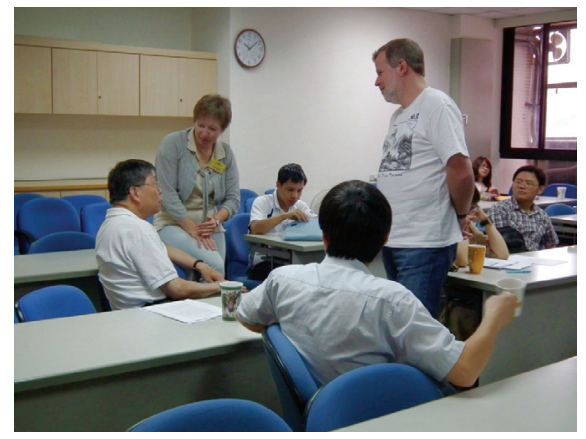

relaxing between talks

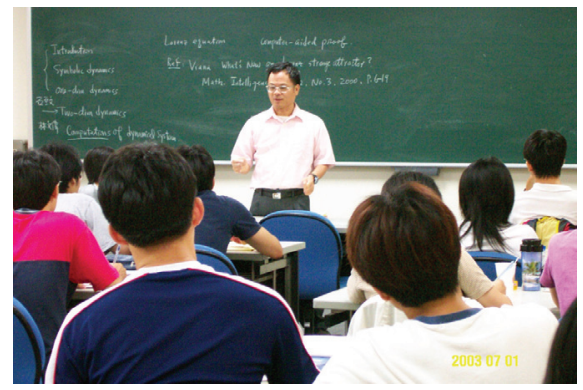

a popular course

(number theory and algebraic geometry) from NTU are Center Scientists at NCTS Taipei. This list is ad- 
justed annually.

Currently in Hsinchu there are 4 postdoctoral fellows (Hau-Wen Huang, Linfeng Mei, Xiaoqing He, and Fang-Ting Tu) and 2 research associates (Chung-Yun Hsieh and HungKung Su). The postdoctoral position is for one year and renewable for another year. Most of the postdoctoral fellows remain in academia after leaving NCTS. The research associates spend one year at NCTS to strengthen their mathematical background and research skills while

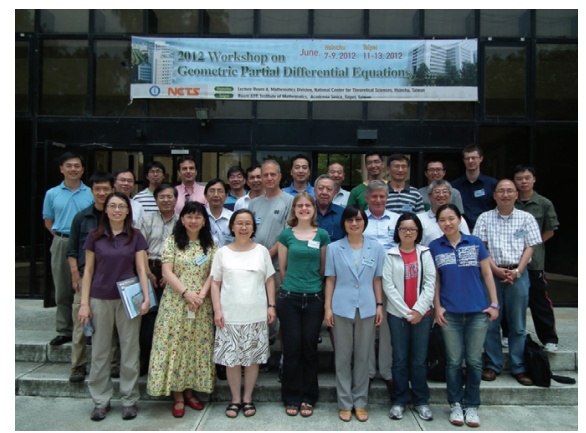

Workshop on Geometric Partial Differential Equations

preparing themselves for studying abroad. This is one of the several ways NCTS help young people to pursue their career in mathematics. NCTS has been quite successful in doing this. Many research associates

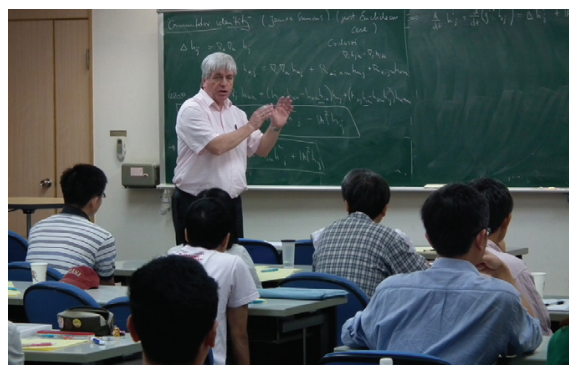

G. Huisken

went to top graduate programs abroad, and some returned to work in various institutions in Taiwan after receiving their Ph.D.

In Taipei, there are 3 postdoctoral fellows (Yi-Chih Chiu, Mounir Hajli, Chung-Jun Tsai) and 2 research associates (Chin-Hung Lin and YuCheng Su).

\section{Administrative Staff}

The division has a team of able administrative staff to provide support and take care of all details related to day-to-day smooth operation of the Center. All visitors are deeply impressed by their efficiency and courtesy. They are: Wendy Huang (黄 雍雅), Vickey Sun (孫毓孜), Chiayu Twu (涂佳玉), Sophie Wang (王寶珍) in Hsinchu; Szuchia Chiu (邱思佳) and

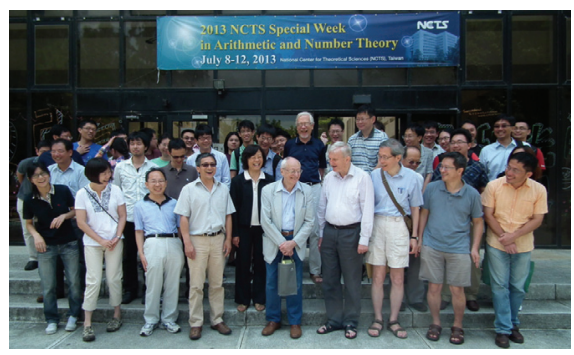

Special Week in Arithmetic and Number Theory

Shu Hui Huang (黃淑惠) in Taipei; and Teresa Pan (潘蒔儀) in Tainan.

\section{Research Programs}

Scientific activities are primarily carried out by director/division head, PI and co-PI of subprojects, center scientists, and focus program coordinators, with assistance from honorary researchers, Chern fellow, and young theorists.

The Mathematics Division has 10 focus programs and 2 regional pro-

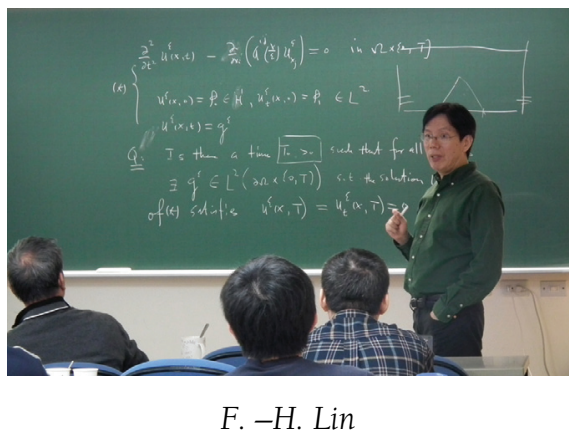

grams. The focus programs and coordinators are as follows:

1. Algebraic Geometry, coordinator: Jungkai Chen

2. Discrete Mathematics, coordin- ators: Gerard Chang and Li-Da Tong

3. Dynamical Systems, coordinator: Kuo-Chang Chen

4. Geometric Analysis and Differential Geometry, coordinators: Jih-Hsin Cheng, Yng-Ing Lee, D-M. Nhieu, Chiung-Jue Sung, DongHo Tsai, and River Chiang

5. Mathematical Biology, coordinators: Sze-Bi Hsu, Chih-Wen Shih, and Je-Chiang Tsai

6. Number Theory, coordinators: Wen-Ching W. Li, Liang-Chung Hsia, Yifan Yang, and Chia-Fu Yu

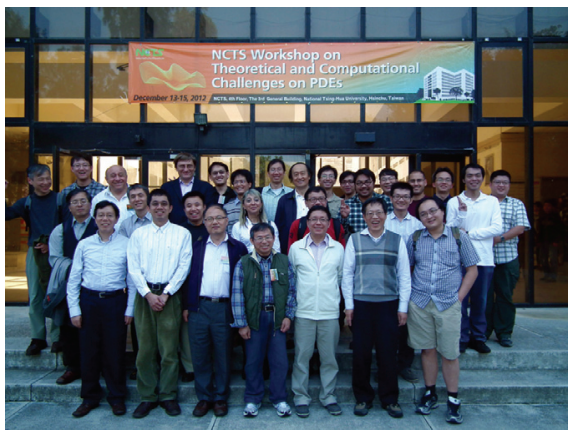

Workshop on Theoretical and Computational Challenges on PDE

7. Partial Differential Equations, coordinators: Jong-Sheng Guo, Tai-Chia Lin, Jenn-Nan Wang, Jann-Long Chern, Yung-Fu Fang, and Chiun-Chuan Chen

8. Probability Theory, coordinators:

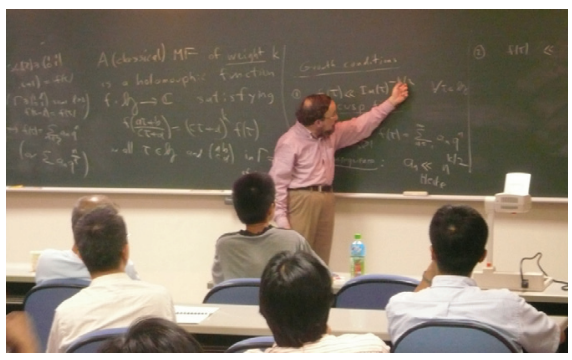

D. Zagier

Shuenn-Jyi Sheu and YuanChung Sheu

9. Representation Theory, coordinators: Shun-Jen Cheng, ChingHung Lam, and Wen-Fong Ke

10. Scientific Computation, coordinators: Ming-Chih Lai, Wen-Wei Lin, Wei-Cheng Wang, Soon-Yi $\mathrm{Wu}$, and I-Liang Chern. 
In addition, there are two regional programs in Statistics, one in Hsinchu coordinated by H. Wang, N. J. Hsu and S.-T. Tseng, and one in Tainan run by Tiee-Jian Wu.

Each focus group determines its own priority and the appropriate scientific activities. At the end of each calendar year, NCTS presents written

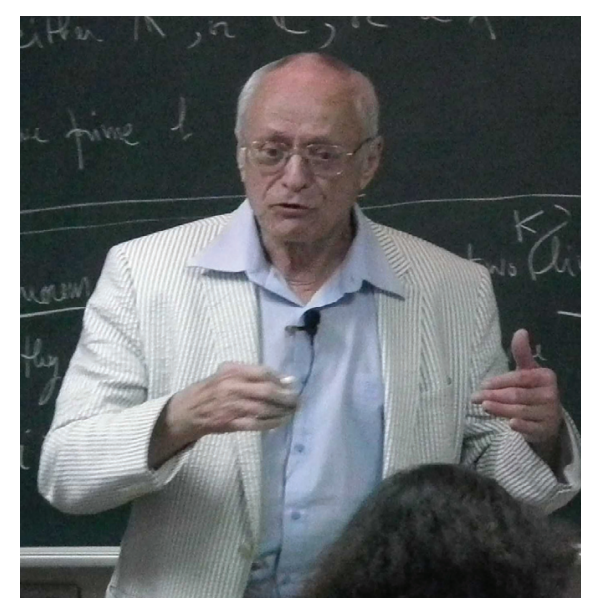

J-P. Serre

and oral reports to the review committee (called by the NSC), which examines the performance of all programs and checks how the funds were used.

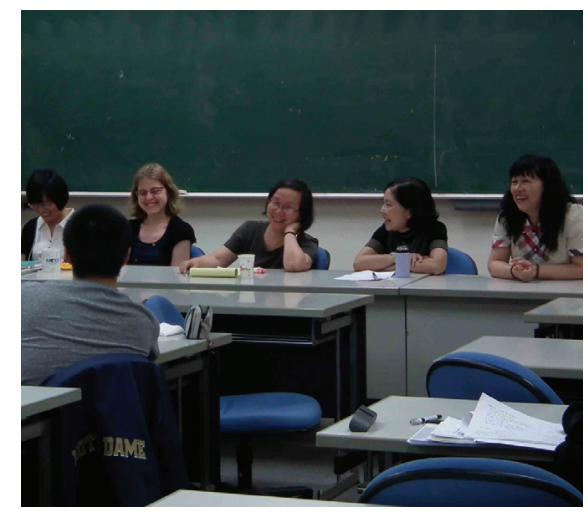

chatting with S.-Y. Chang and female mathematicians

\section{Highlights of Activities}

The Center is a lively and intellectually stimulating place for all, from undergraduate students to senior researchers. We offer regular seminars and winter/summer schools to train undergraduate and graduate students, weekly research seminars for graduate students, postdoctoral fellows, and faculty members. Continuous efforts are made to invite domestic and international experts to offer special topic courses designed to discuss cutting edge research progress, aiming to identify research topics and start research collaborations. The numerous visitors report on their recent advances in diverse areas of mathematics. Each focus program organizes at least one workshop/ conference in its field every year to showcase results by Taiwanese researchers and to provide a platform for domestic researchers to interact

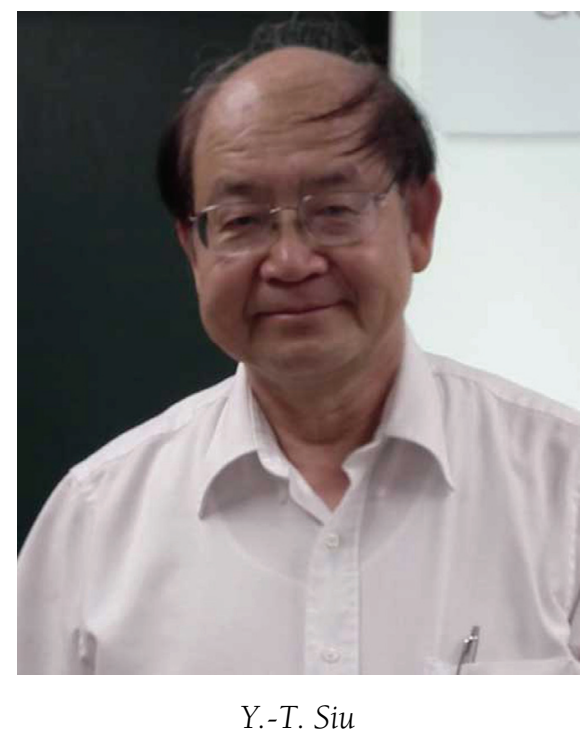

with the international experts. In recent years mathematicians and physicists have jointly organized workshops and summer schools to promote further communications between the two divisions of the Center.

The special year in the Mathematics Division was initiated in 2010. The purpose is to allocate more resources to a targeted area to give it a strong push. The themes are: number theory (2010), geometry (2011), applied mathematics (2012), algebraic geometry and representation theory (2013), and number theory (2014). It has been very fruitful. As a result, interactions and collaborations among people from different insti- tutions have remarkably increased, and through jointly organized inter-disciplinary and cross-area activities, people have broadened their horizon.

The "Distinguished Lecture Series" are the most prestigious lectures in the Mathematics Division. Its inaugural speaker was Jean-Pierre

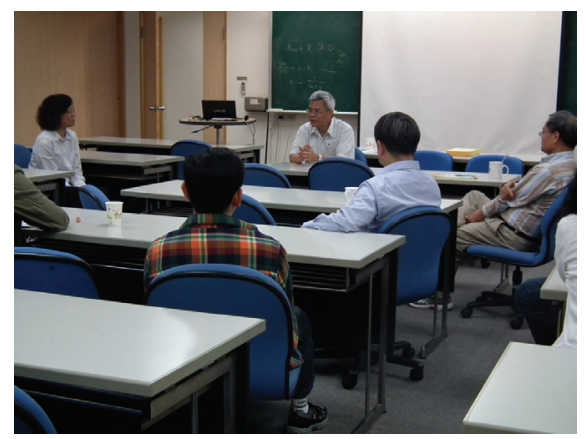

chatting with C. - S. Lin

Serre of College de France in 2009. Beginning 2010, we incorporate this lecture series with activities of the special year. We are very fortunate that Don Zagier of Max Planck Mathematics Institute, Bonn, and College de France (2010), Gerhard

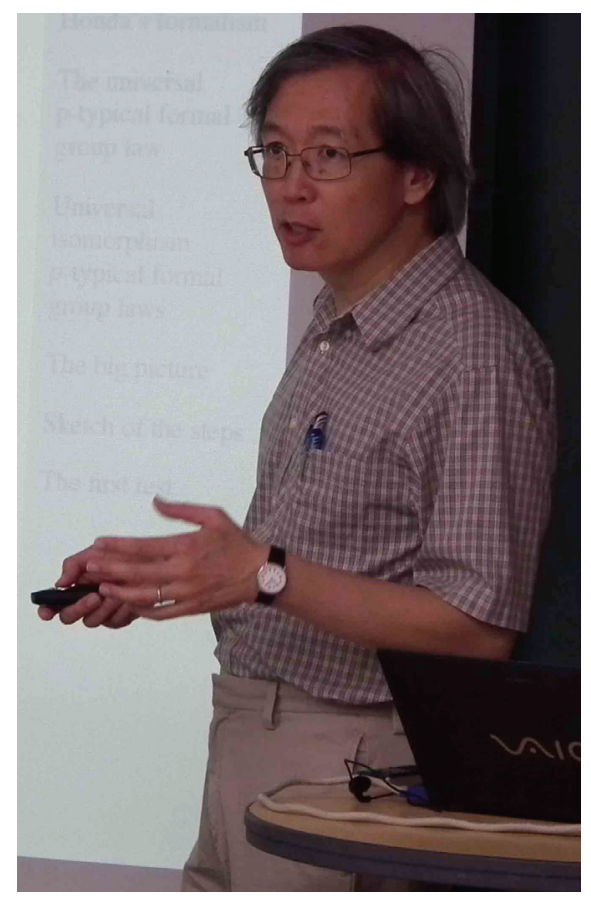

C. - L. Chai

Huisken of Max Planck Institute of Gravitational Physics (2011), FangHua Lin of Courant Institute of 
Mathematical Sciences (2012), JeanPierre Serre of College de France (2013), and Manjual Bhargava of Princeton University (2014) have accepted our invitation to deliver the Distinguished Lecture Series.

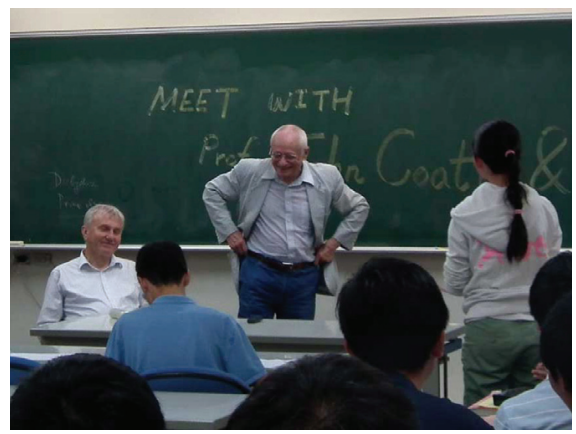

chatting with John Coates and J-P. Serre

In 2012 the Mathematics Division launched a new "Meet with Professor..." series to devote one day to feature a famous mathematician. Activities include lectures to highlight his/her research achievements and a forum discussion to show his/her

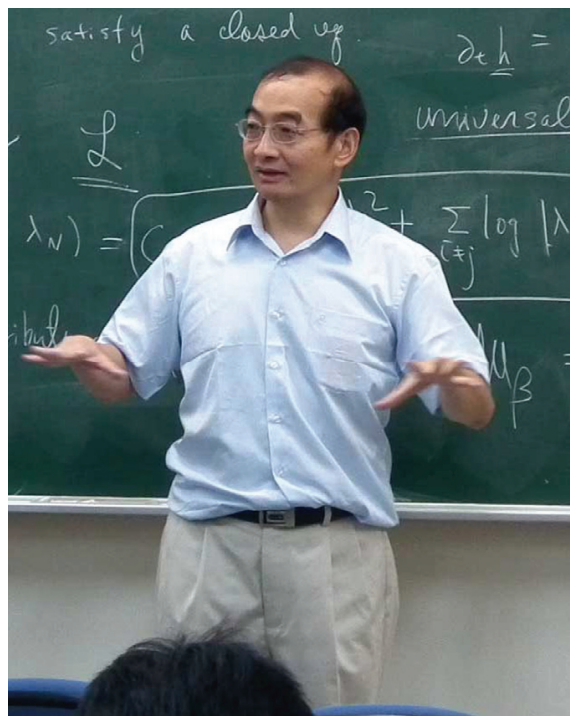

H.-T. Yau

human side. The purpose is for the audience to learn from very successful people their mathematics and how to handle challenges in mathematics. We have featured Sun-Yung Alice Chang of Princeton University, Yum-Tong Siu of Harvard University, Ching-Li Chai of University of Pennsylvania and Academia Sinica, and Chang-Shou Lin of National Taiwan

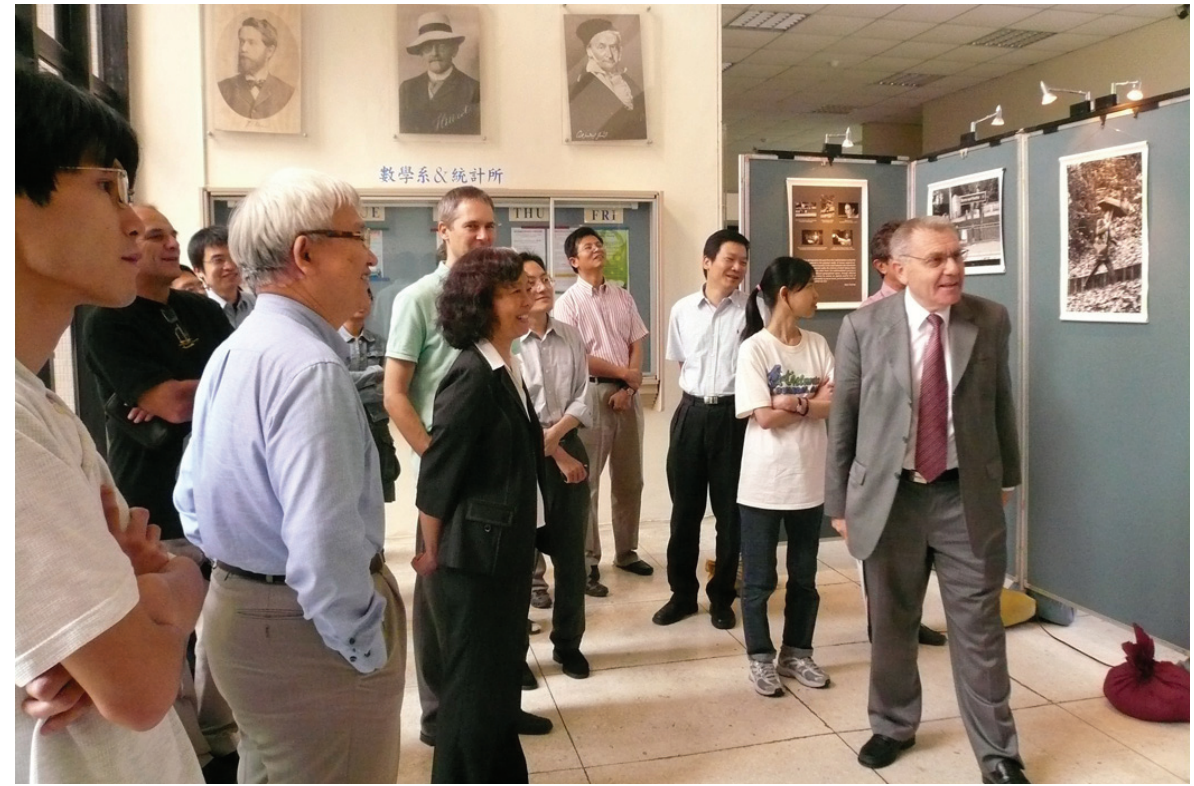

photo exhibition from I.H.E.S., France

University in 2012, and Jean-Pierre Serre of College de France, John Coates of University of Cambridge, Horng-Tzer Yau of Harvard University, and Jing Yu of National Taiwan University in 2013.

To promote international cooperation and collaboration is one of our main goals. In recent years, the
Mathematics Division has signed MOU with prestigious institutions in the world. These include: Pohang Mathematics Institute, Korea (2010), Russian-French Poncelet Lab, Russia (2011), Independent University of Moscow, Russia (2011), University of Bergen, Norway (2012), Tsinghua University Mathematical Sciences Center, China (2013). There have

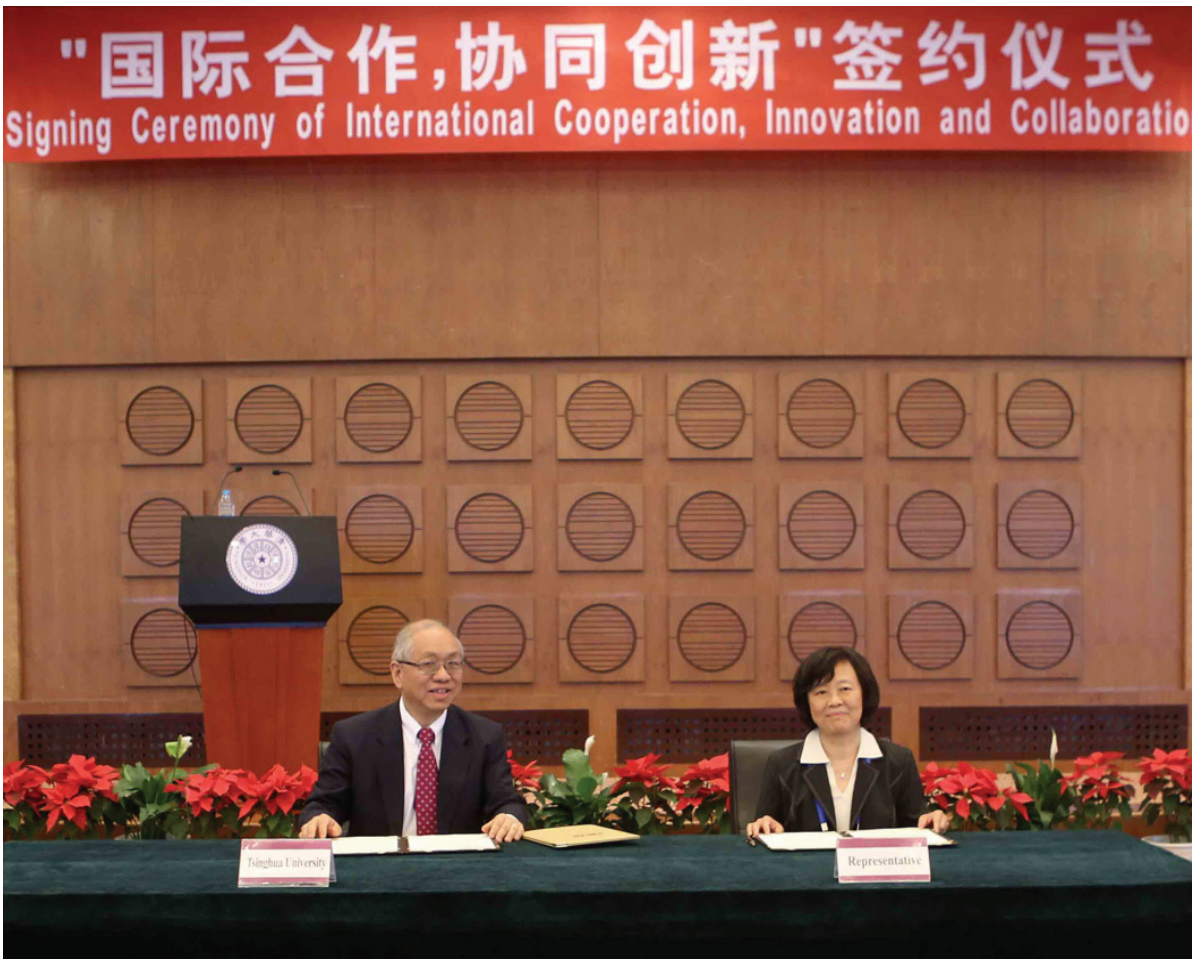

signing MOU with MSC of Tsinghua Univ., China 
been regular exchanges between NCTS and the MOU partners. The most frequent one is the rotating annual workshop in number theory and PDE between PMI and NCTS since 2009. There have been biannual conferences in number theory (resp. PDE) between NCTS and Waseda (resp. Tohoku) University in Japan. Beginning 2012, Taiwan has become a member country of the Pan Asian Number Theory community, with Wen-Ching Li serving on its Scientific Committee representing Taiwan. These agreements provide more opportunities to elevate the visibility and enhance the quality of research of Taiwanese mathematicians. They have also resulted in international collaborations.

The Center also seeks cooperation with international funding agencies. We have successfully organized three conferences jointly supported by NCTS and the National Science Foundation in US: Workshop on PDE Model: Biological Process (2010), Workshop on Fluid-Structure Interaction Problems (2011), International Conference on Galois Representations, Automorphic Forms, and Shimura Varieties (2011). This kind of international cooperation was first in Taiwan.

\section{Awards and nurturing young talents}

The foremost mission of NCTS is to nurture young talents. A substantial portion of our funds is used for this purpose. We strongly encourage young researchers, including Ph.D. students, postdoctoral fellows, and junior faculty members to attend important programs and conferences abroad or spend an extended period of time to work with international experts. Those interested can apply for CTS fellows to support such activities. This has been an important and effective way to foster young generations. Many people have benefited from this program.

Each year NCTS offers Young Theorists Awards to young faculty members. The recipients in mathematics this year are Ming-Lun Hsieh of NTU (number theory), and
Ching-Hsiao Cheng of NCU (nonlinear PDE); together with Chieh-Yu Chang of NTHU (number theory) and
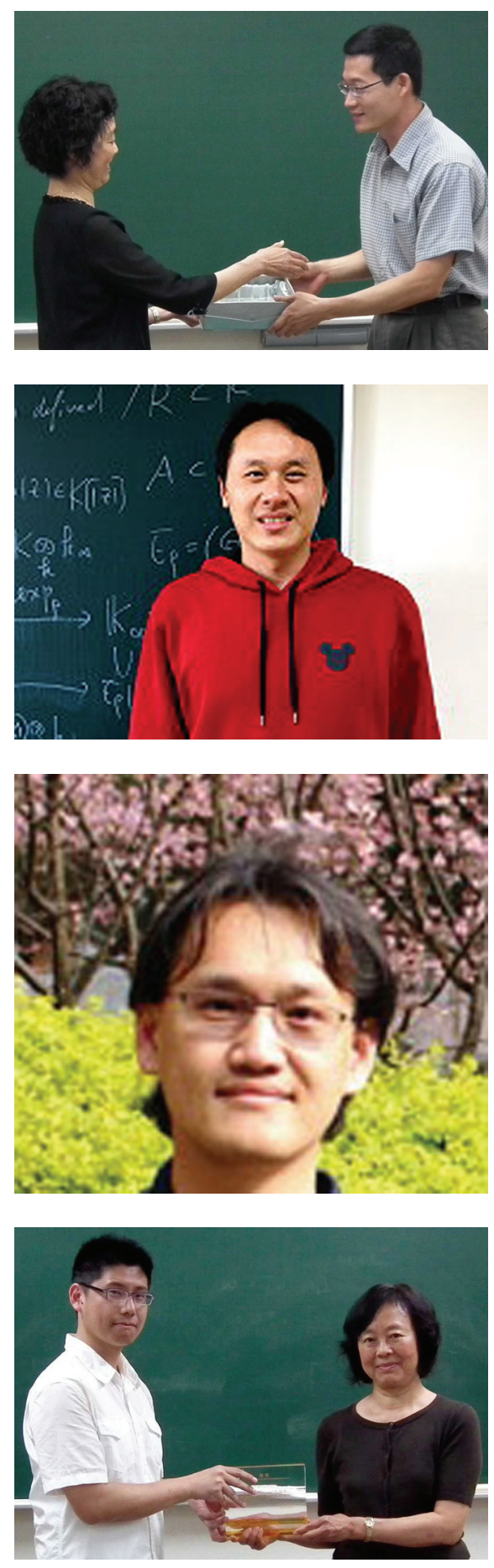

NCTS Young Theorists.

Photos from top: J.-C. Tsai (at right),

C.-Y. Chang, C.-H. Cheng, and M.-L. Hsieh (at left)

Je-Chiang Tsai of NCCU (mathematical biology), there are four young theorists at present. We are very proud that in the past decade all NSC 吳大猶 Award winners in mathematics have been NCTS young theorists. Kuo-Chang Chen of NTHU (dynamical systems) is the current Chern Fellow (2010-14), the most prestigious award of the Mathematics Division. The honorary researcher award aims to solicit well-established and highly active mathematicians to help run activities at the Center. Chia-Fu $\mathrm{Yu}$ of AS was the most recent NCTS honorary researcher (2012-13).

Mathematicians active in NCTS have won important international and domestic recognitions in recent years. International awards include:

- ICCM Morningside silver medal: Chin-Lung Wang of NTU (2001), Chiun-Chuan Chen of NTU (2007), Jung-Kai Chen of NTU (2010), Chieh-Yu Chang of NTHU (2013);

- ICCM Chern award: Song-Sun Lin of NCTU (2001), Wen-Ching Li of NCTS (2010);

- $\quad$ AMS inaugural fellow: Wen-Ching Li of NCTS (2012), Jing Yu of NTU (2012);

- SIAM fellow: Sze-Bi Hsu of NTHU (2013).

Within Taiwan, the former director Jing Yu of NTU was elected Academician of Academia Sinica in 2012; Chiun-Chuan Chen of NTU, Chin-Lung Wang of NTU, Wen-Wei Lin of NCTU, and Sze-Bi Hsu of NTHU were named the prestigious Ministry of Education National Chair Professor (教育部國家講座); Gerard Chang of NTU, Meng-Kiat Chuah of NTHU, Shun-Jen Cheng of AS, and Jung-Kai Chen of NTU received Ministry of Education Outstanding Research Award (教育部學術獎). The NSC distinguished award recipients in the past decade are all avid participants of NCTS, many of them benefited greatly from NCTS. Among them Chieh-Yu Chang of NTHU is the only assistant professor receiving this honor. C.-Y. Chang was a postdoctoral fellow at NCTS for four years before joining NTHU. 


\section{The Impact of NCTS}

In the past 15 years, the research in theoretical sciences in Taiwan has improved remarkably. It is generally believed that the superb job done by NCTS played the pivotal role. Some statistical data support the above statement. In the year before 1993, the total number of articles in mathematics in SCI journals is less than 100. The quantity grew dramatically after 1998, one year after the founding of NCTS. Not only the quantity, but also the overall quality of the research work is improved strikingly as well. Figure 1 below (based on information from Essential Science Indicator) compares the recent data on citations per mathematics paper from Taiwan with neighboring countries, including Korea, China, USA, and world average. It shows that we are now a little above the world average, China, and Korea.

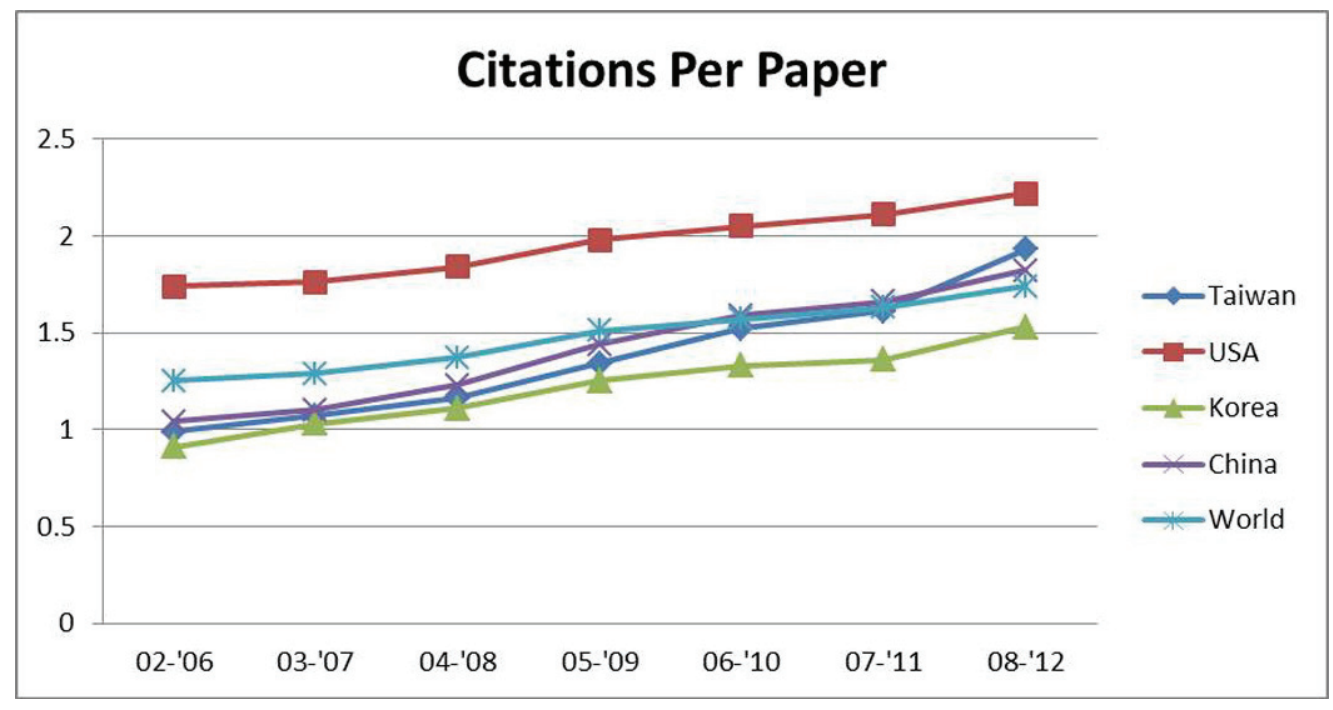

Figure 1. Citations per paper.

Table 1 below shows the growth of publications in high quality journals in pure math., applied math., and statistics. We list the number of papers ranked $A^{*}$ (= top 7\%) and A (next 19\%) according to ERA journal ratings for mathematics, total means the number of all papers in that category.

\begin{tabular}{|c|c|c|c|c|c|c|c|c|c|c|c|c|c|}
\hline \multicolumn{2}{|l|}{ year } & 2001 & 2002 & 2003 & 2004 & 2005 & 2006 & 2007 & 2008 & 2009 & 2010 & 2011 & 2012 \\
\hline \multirow{3}{*}{$\begin{array}{l}\text { NCTS as } \\
\text { affiliation }\end{array}$} & $\mathrm{A}^{*}$ & 0 & 1 & 1 & 0 & 2 & 3 & 1 & 5 & 10 & 17 & 12 & 13 \\
\hline & $\mathrm{A}$ & 1 & 0 & 0 & 1 & 4 & 8 & 10 & 27 & 18 & 17 & 17 & 33 \\
\hline & total & 1 & 1 & 1 & 3 & 10 & 16 & 28 & 52 & 54 & 81 & 53 & 90 \\
\hline \multirow{3}{*}{$\begin{array}{l}\text { acknowledging } \\
\text { NCTS }\end{array}$} & $\mathrm{A}^{*}$ & 1 & 1 & 0 & 0 & 5 & 10 & 10 & 15 & 19 & 20 & 17 & 18 \\
\hline & $\mathrm{A}$ & 1 & 0 & 0 & 2 & 8 & 9 & 16 & 22 & 24 & 27 & 24 & 30 \\
\hline & total & 2 & 2 & 4 & 5 & 25 & 33 & 43 & 57 & 108 & 114 & 74 & 95 \\
\hline \multirow[t]{3}{*}{ other } & $\mathrm{A}^{*}$ & 1 & 0 & 0 & 0 & 0 & 1 & 2 & 1 & 2 & 0 & 0 & 0 \\
\hline & $\mathrm{A}$ & 0 & 0 & 0 & 0 & 0 & 0 & 1 & 6 & 0 & 0 & 0 & 0 \\
\hline & total & 1 & 0 & 0 & 0 & 11 & 3 & 7 & 16 & 23 & 0 & 0 & 0 \\
\hline
\end{tabular}

Table 1. Number of high-quality papers.

This is presented graphically by Figure 2 below. 


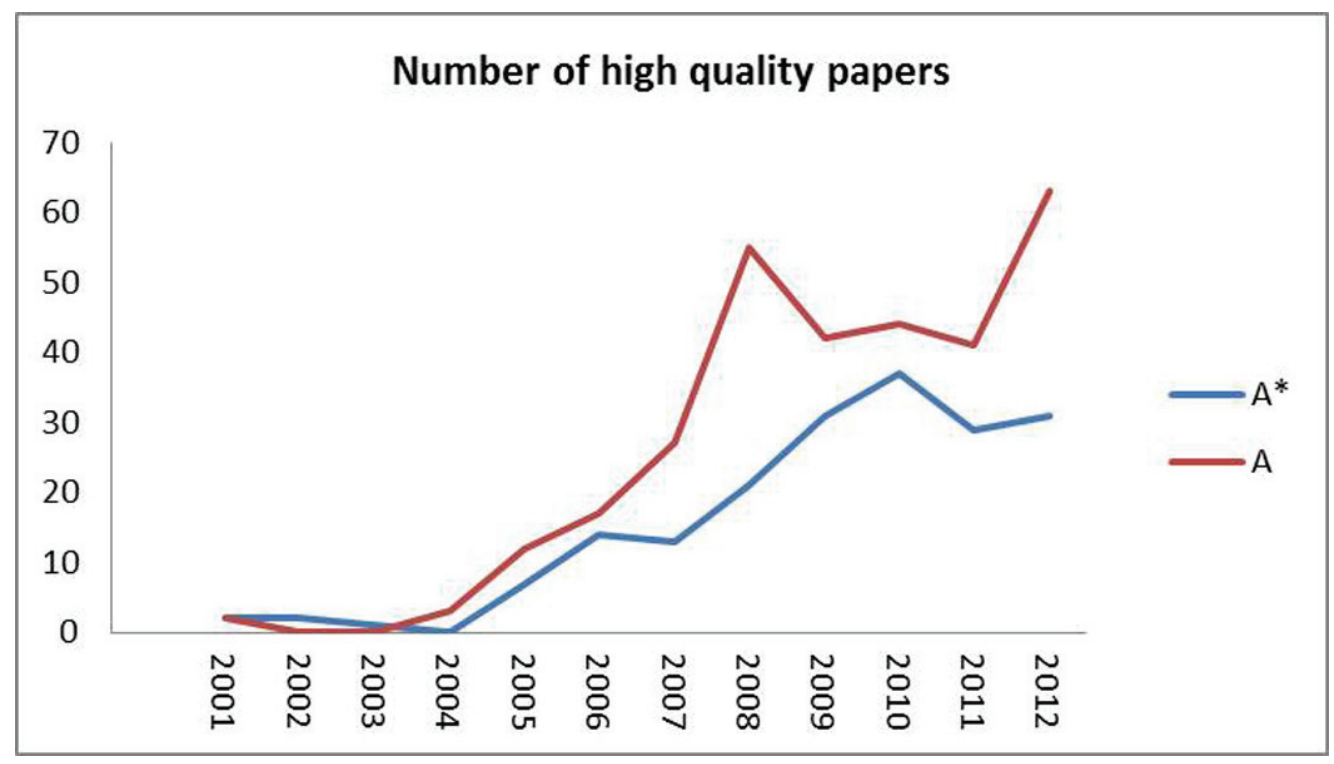

Figure 2. Number of high-quality papers.

Figure 3 below shows the international standing of mathematics in Taiwan measured by the impact relative to field from 1981 to 2010. Relative impact 1 means world average.

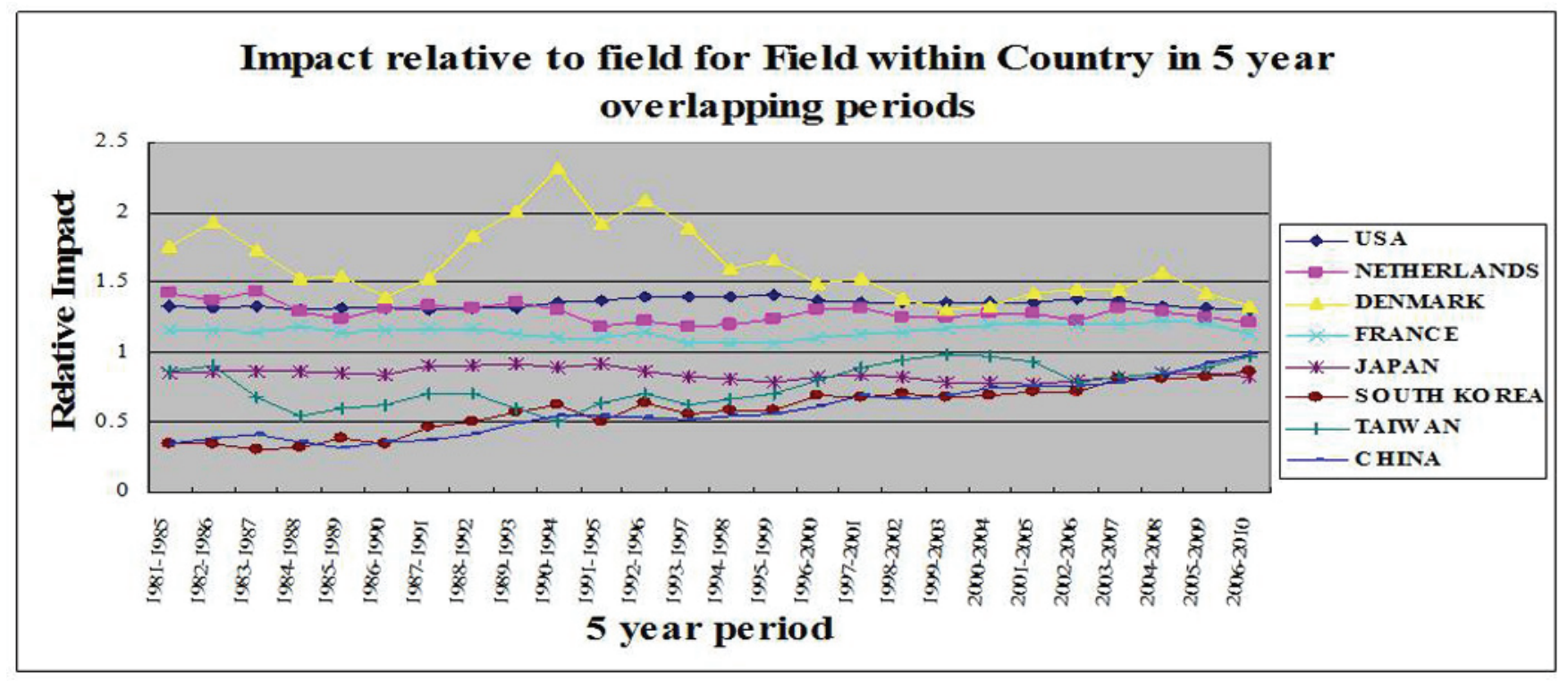

Figure 3. Relative impact over 5 years, by country.

Information source: National Science Indicators on Diskette. 2010 information updated 10 January 2012.

2006-2010 relative impact factors: Denmark (1.33), USA (1.3), Netherlands (1.21), France (1.13), China (0.98), Taiwan (0.97), South Korea (0.86), Japan (0.82).

Figure 4 below compares the performance of mathematics relative to other subject areas within natural sciences in Taiwan from 1981 to 2010. It shows that mathematics performs best among natural sciences in Taiwan. 


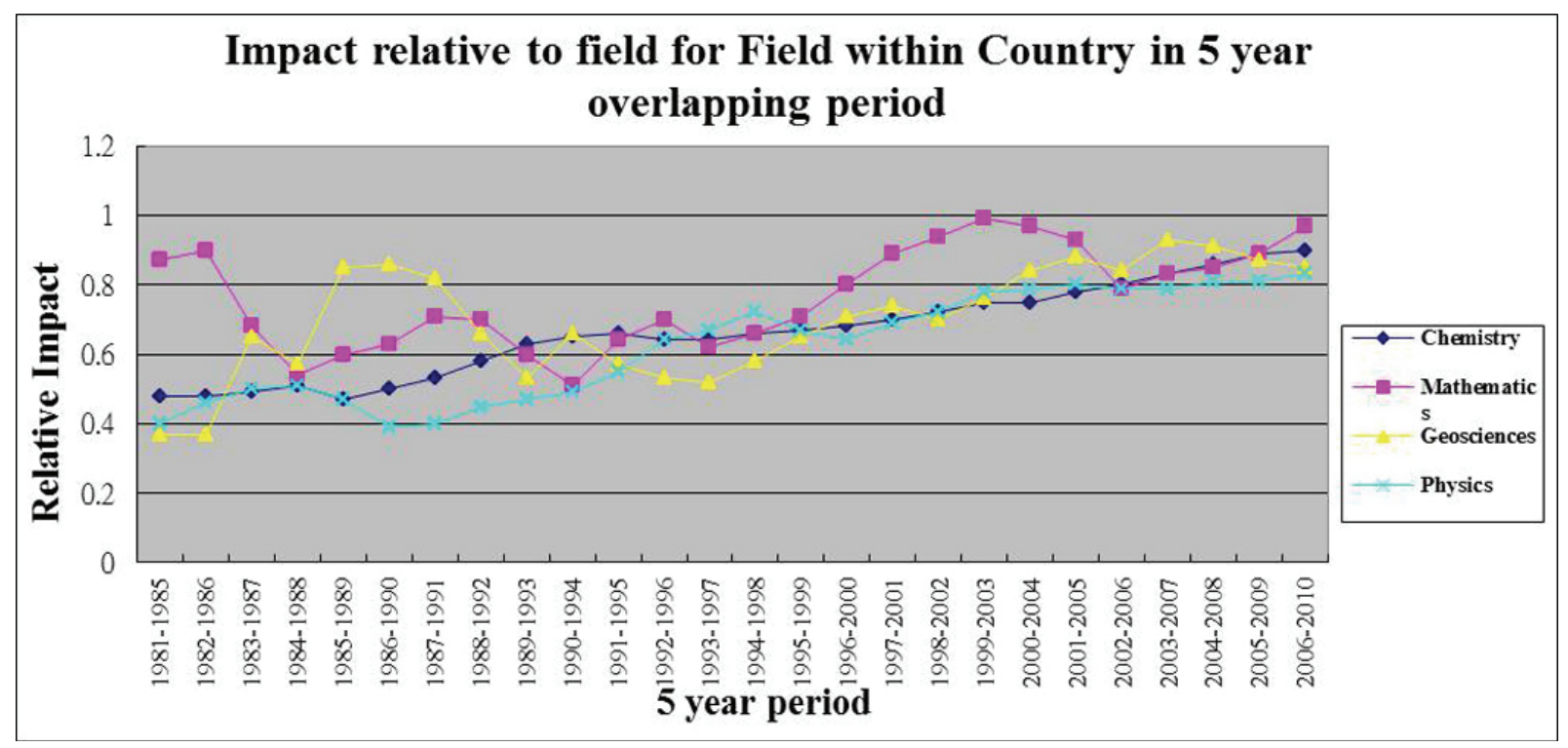

Figure 4. Relative impact over 5 years, in Taiwan, by subject area.

Information source: National Science Indicators on Diskette. 2010 information updated 10 January 2012.

2006-2010 relative impact factors: Mathematics (0.97), Chemistry (0.90), Geosciences (0.85), Physics (0.83). below.

In terms of support (in million TWD) and performance, mathematical sciences are most cost-effective. See Figure 5

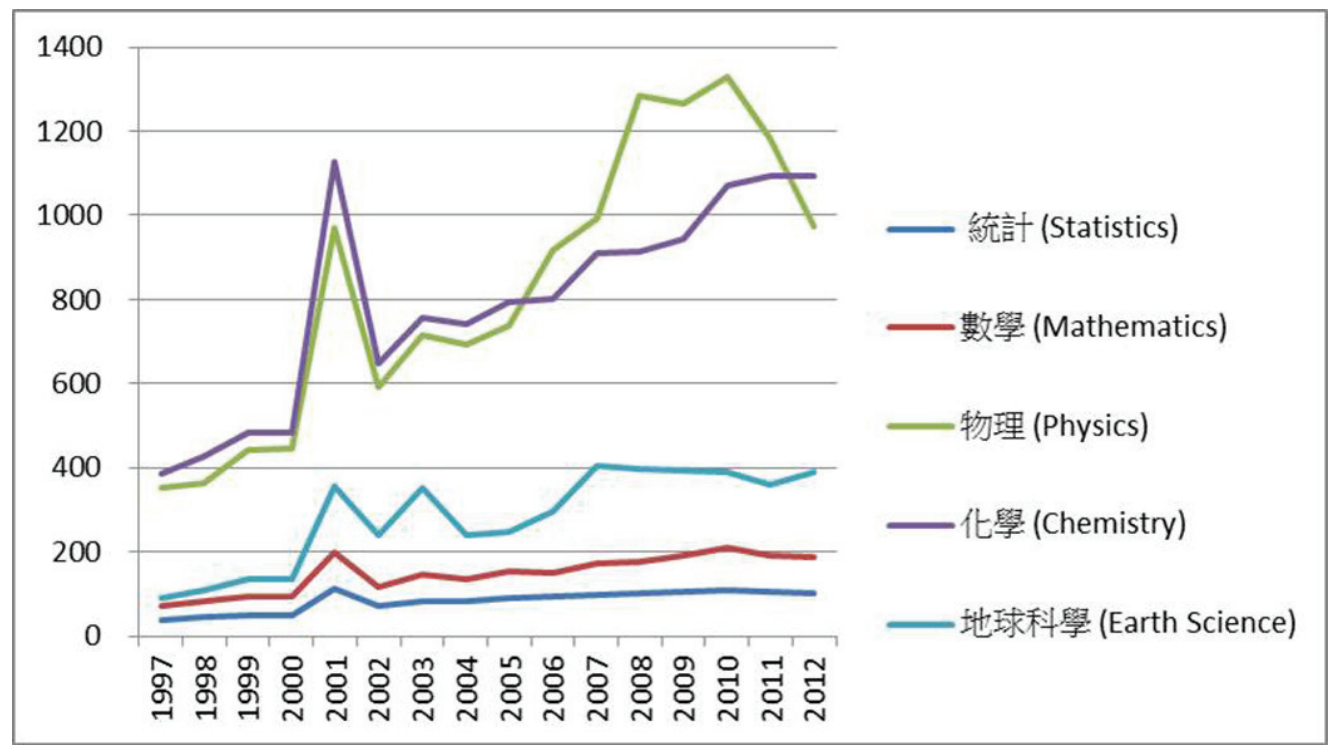

Figure 5. 